\title{
Apolipoprotein D alleviates glucocorticoid- induced osteogenesis suppression in bone marrow mesenchymal stem cells via the PI3K/Akt pathway
}

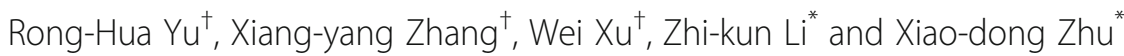

\begin{abstract}
Background: To clarify the role of apolipoprotein D (Apod) in alleviating glucocorticoid-induced osteogenesis suppression in bone marrow mesenchymal stem cells (MSCs) via the PI3K/Akt pathway, thus influencing the progression of osteoporosis (OP).

Methods: Osteogenesis in MSCs was induced by dexamethasone (DEX) stimulation. Dynamic expressions of Apod in MSCs undergoing osteogenesis for different time points were determined by qRT-PCR. Relative levels of osteogenesis-associated genes, including ALP, RUNX2, and Osterix, in DEX-induced MSCs overexpressing Apod or not were examined. Moreover, the protein level of RUNX2, ALP, and Osterix; ALP activity; and mineralization ability influenced by Apod in osteogenic MSCs were assessed. At last, the potential influences of Apod on the PI3K/Akt pathway were identified through detecting the expression levels of PI3K and Akt in MSCs by Western blot.

Results: Apod was time-dependently upregulated in MSCs undergoing osteogenesis. DEX induction downregulated ALP, RUNX2, and Osterix and attenuated ALP activity and mineralization ability in MSCs undergoing osteogenesis, which were partially reversed by overexpression of Apod. In addition, Apod overexpression upregulated the reduced levels of PI3K and Akt in DEX-induced MSCs.

Conclusion: Apod alleviates glucocorticoid-induced osteogenesis suppression in MSCs via the PI3K/Akt pathway, thus protecting the progression of OP.
\end{abstract}

Keywords: Apod, MSCs, PI3K/Akt, Osteogenesis

\section{Background}

Glucocorticoid-induced osteoporosis (GIOP) is a common disease following long-term use or large doses of glucocorticoids [1, 2]. GIOP enhances susceptibility to bone fractures [3]. Attenuated bone formation and accelerated bone resorption lead to imbalanced progression, which is

\footnotetext{
* Correspondence: scoliosis_lizk@126.com; scoliosis@126.com

${ }^{+}$Rong-Hua Yu, Xiangyang Zhang, and Wei Xu contributed equally to this article and should be listed as co-first authors.

Department of Orthopedics, Tongren Hospital, School of Medicine, Shanghai JiaoTong University, 1111 Xianxia Road Shanghai, Shanghai 200336, P.R. China
}

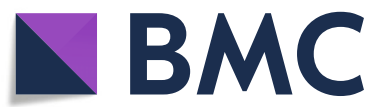

(c) The Author(s). 2020 Open Access This article is licensed under a Creative Commons Attribution 4.0 International License, which permits use, sharing, adaptation, distribution and reproduction in any medium or format, as long as you give appropriate credit to the original author(s) and the source, provide a link to the Creative Commons licence, and indicate if changes were made. The images or other third party material in this article are included in the article's Creative Commons licence, unless indicated otherwise in a credit line to the material. If material is not included in the article's Creative Commons licence and your intended use is not permitted by statutory regulation or exceeds the permitted use, you will need to obtain permission directly from the copyright holder. To view a copy of this licence, visit http://creativecommons.org/licenses/by/4.0/. The Creative Commons Public Domain Dedication waiver (http//creativecommons.org/publicdomain/zero/1.0/) applies to the data made available in this article, unless otherwise stated in a credit line to the data.

the major pathogenic reason for osteoporosis. Some drugs are reported to induce the occurrence of OP [4]. Among them, GIOP is prevalent. Even the administration of physiological doses of glucocorticoid (GC) can lead to bone loss. Menopausal women and men over 50 years of age are the risk population of OP. In particular, patients with joint diseases with long-term, continuous GC treatment are the highest-risk population of OP [4].

Apolipoprotein D (ApoD) is a $29-\mathrm{kDa}$ glycoprotein highly conserved lipocalin known for its antioxidant and neuroprotective functions [5]. Unlike other apolipoproteins, 
they are essentially produced in the liver and intestine. Previous studies reported that ApoD was upregulated in many nervous system diseases (Alzheimer's disease [6], Parkinson's disease [7], and stroke [8]). Martineau et al. [9] reported that ApoD deficiency is associated with high bone turnover, low bone mass, and impaired osteoblastic function in aged female mice. Ishii et al. [10] found that ApoD gene expression was increased after osteogenic differentiation. Similar observations were reported in the murine MSC-like cell line C3H10 [11] and in mouse primary osteoblasts [12].

Studies have found that bone cells can participate in the regulation of biological activities of osteoblasts and osteoclasts through activating certain pathways [13]. Activation of the PI3K/Akt pathway in bone cells has been demonstrated to accelerate bone formation by stimulating osteogenesis and osteoblast resorption [14]. Meanwhile, it positively mediates osteogenesis in mesenchymal stem cells (MSCs) $[15,16]$. PI3K/Akt was required for chondrocyte and osteoblast differentiation, and inhibition of PI3K/Akt could inhibit longitudinal bone growth [17].

This paper mainly analyzed the potential function and potential mechanism of Apod in affecting osteogenic differentiation of MSC osteogenesis.

\section{Materials and methods}

\section{Cell culture and osteogenesis induction}

MSCs were purchased from Fuyuan Bio (Shanghai, China) that were isolated from two donors and cultured in low-glucose DMEM (Gibco) supplemented with 10\% FBS (Gibco) and 1\% penicillin/streptomycin and cultured in a humidified incubator at $37{ }^{\circ} \mathrm{C}$ and $5 \% \mathrm{CO}_{2}$. Cell passage was conducted every 2 days. For osteogenesis induction, MSCs were inoculated in a 6-well plate at a density of $2.0 \times 10^{5} / \mathrm{mL}$. Osteogenic induction was applied as follows: DMEM $+10 \%$ FBS $+1 \%$ penicillinstreptomycin $+10^{-7} \mathrm{~mol} / \mathrm{L} \mathrm{DEX}+10 \mathrm{mmol} / \mathrm{L} \beta$-glycerophosphate $+50 \mu \mathrm{g} / \mathrm{mL}$ ascorbic acid. Osteogenesis was induced for 7-14 days. The medium was replaced every 3 days.

\section{DEX treatment}

Cell suspension was prepared at a density of $5 \times 10^{4} / \mathrm{mL}$. A total of $100 \mu \mathrm{L}$ (containing $5 \times 10^{3}$ cells) of suspension was applied in each well of a 96-well plate. After overnight cell adherence, medium containing $1 \mu \mathrm{M}$ DEX was replaced.

\section{Cell transfection}

Cell transfection was conducted using Lipofectamine 2000 (Invitrogen, Carlsbad, CA, USA). Transfection vectors were provided by GenePharma (Shanghai, China).
MSCs were infected with the Apod-expressing lentivirus or control lentivirus in the presence of $8 \mu \mathrm{g} / \mathrm{mL}$ polybrene, and the resulting cell lines were named MSCApod and MSC-GFP, respectively.

\section{Quantitative reverse transcriptase polymerase chain reaction assay (q-RTPCR) analysis}

Total RNA was extracted using TRIzol reagent (Invitrogen, Carlsbad, CA, USA). Total RNA was quantified by measuring the absorbance at $260 \mathrm{~nm}$ (NanoDrop 2000; Thermo Fisher Scientific, MA, USA). Single-strand cDNA was synthesized by reverse transcription with a Superscript II Reverse Transcriptase Kit and oligo dT primers (Invitrogen, Carlsbad, CA, USA) and amplified by reverse transcription PCR with iQ SYBR Green Supermix (Biorad, Hercules, CA, USA). qPCR was performed using SYBR Green PCR Master Mix (Applied Biosystems), following the manufacturer's instructions. The expression levels of the target genes were calculated relative to housekeeping GAPDH using Stratagene Mx3000P software (Applied Biosystems) with the $2^{-\Delta \Delta C t}$ equation. The primer sequences were listed in Table 1.

\section{Western blot}

The total proteins were extracted by radioimmunoprecipitation assay (RIPA) (Solarbio, Beijing, China) lysate and then separated by sodium dodecyl sulfatepolyacrylamide gel electrophoresis (SDS-PAGE). The separated proteins were transferred onto the polyvinylidene difluoride membranes. The membranes were blocked with 5\% nonfat milk dissolved in Trisbuffered saline with $0.05 \%$ Tween-20 (TBS-T) for $1 \mathrm{~h}$ at room temperature. The membranes were then incubated overnight at $4{ }^{\circ} \mathrm{C}$ with primary antibodies against Apod (1:2000), Osterix (1:1000), ALP (1:4000), RUNX2 (1:2000), p-PI3K (1:2000), PI3K(1:1000), pAkt (S437, 1:2000), Akt (1:2000), and GAPDH (1: 8000 ). Finally, the membranes were incubated for $1 \mathrm{~h}$ with horseradish peroxidase (HRP)-conjugated secondary antibodies (1:500) and visualized using an enhanced chemiluminescence system, according to the manufacturer's instructions.

Table 1 Sequences of primers for quantitative real-time PCR

\begin{tabular}{lll}
\hline Gene & Forward primer (5'-3') & Reverse primer (3'-5') \\
\hline GAPDH & TTCTTITGCGTCGCCAGCCGA & GTCACCACCCGCCCAATACGA \\
RUNX2 & GGGTAAGACTGGT-CATAGGACC & CCCAGT-ATGAGAGTAGGTGTCC \\
ALP & ACCACCACGAGAGTGAACCA & CGTTGTCTGAGTACCAGTCCC \\
Osterix & AGGAGGCACAAAGAAGCCATAC & AGGGAAGGGTGGGTAGTCATT \\
Apod & TTAACCTCACAGAGCCTGCC-3 & GAGTCCACTGTTCTGGAGGG \\
\hline
\end{tabular}




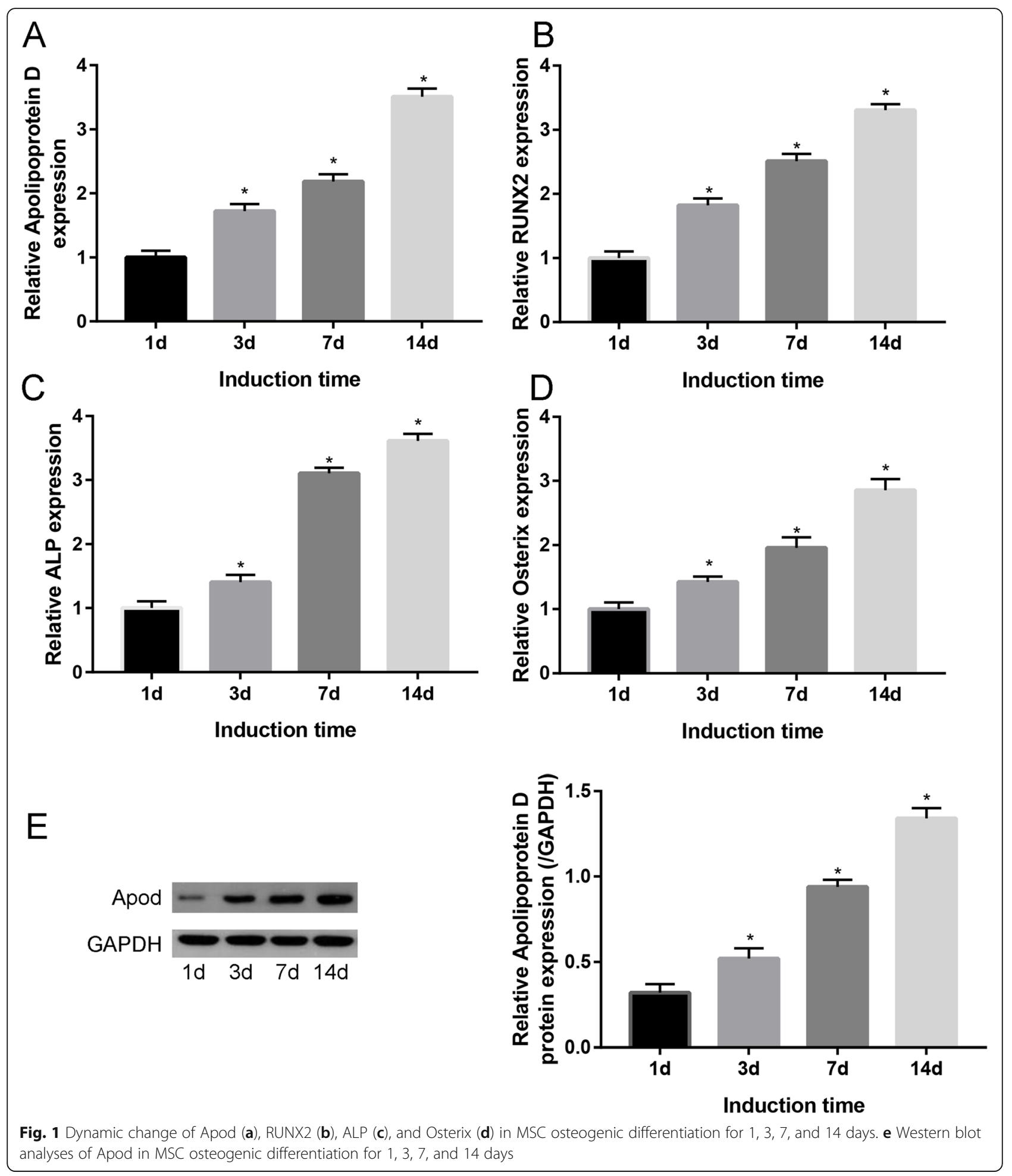

Alkaline phosphatase (ALP) activity determination

MSCs were washed with pre-cold PBS for three times and lysed in pre-cold $1 \%$ Triton X-100 on ice for $30 \mathrm{~min}$. The cell lysate was subjected to ALP activity determination, and the value at $405 \mathrm{~nm}$ was normalized to that of total protein concentration.

\section{ALP staining}

MSCs were washed with PBS twice and reacted in 70\% ethanol for $10 \mathrm{~min}$ and ALP buffer $(0.15 \mathrm{M} \mathrm{NaCl}, 0.15 \mathrm{M}$ Tris$\mathrm{HCl}, 1 \mathrm{mM} \mathrm{MgCl}$, pH 9.5) for $15 \mathrm{~min}$. Subsequently, cells were cultured in the NBT-BCIP solution at $37^{\circ} \mathrm{C}$, in the dark for $30 \mathrm{~min}$. Images were captured under a microscope. 


\section{Alizarin red staining (ARS)}

MSCs were induced for 14-day osteogenesis. Cells were washed, fixed in 95\% ethanol for $14 \mathrm{~min}$, and dyed in $0.1 \%$ ARS-Tris-HCL solution ( $\mathrm{pH} 4.3$ ). Visible mineralized nodules were captured under an inverted microscope.

\section{Bioinformatic analysis}

To reveal the potential mechanism of Apod, the Search Tool for the Retrieval of Interacting Genes (STRING, http://string-db.org) database was searched the adjacent molecular of Apod. The Molecular Complex Detection (MCODE) app was used to analyze protein-protein
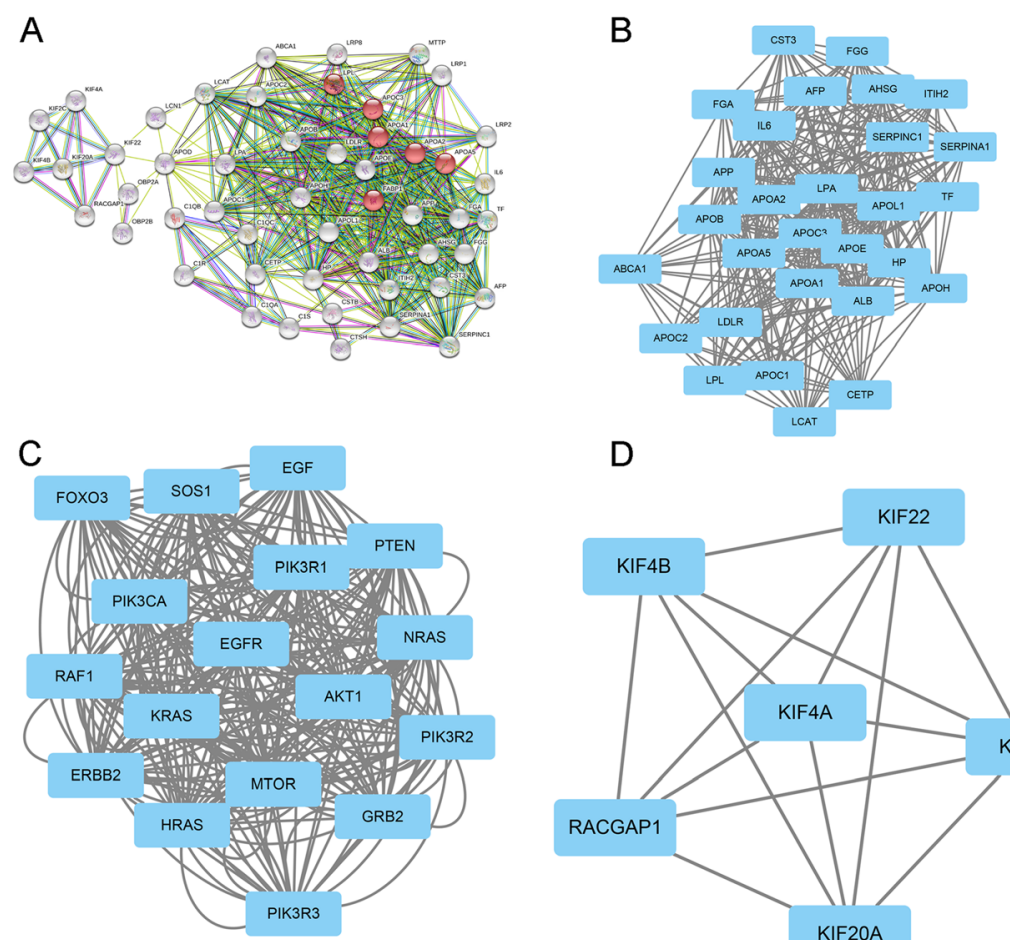

E
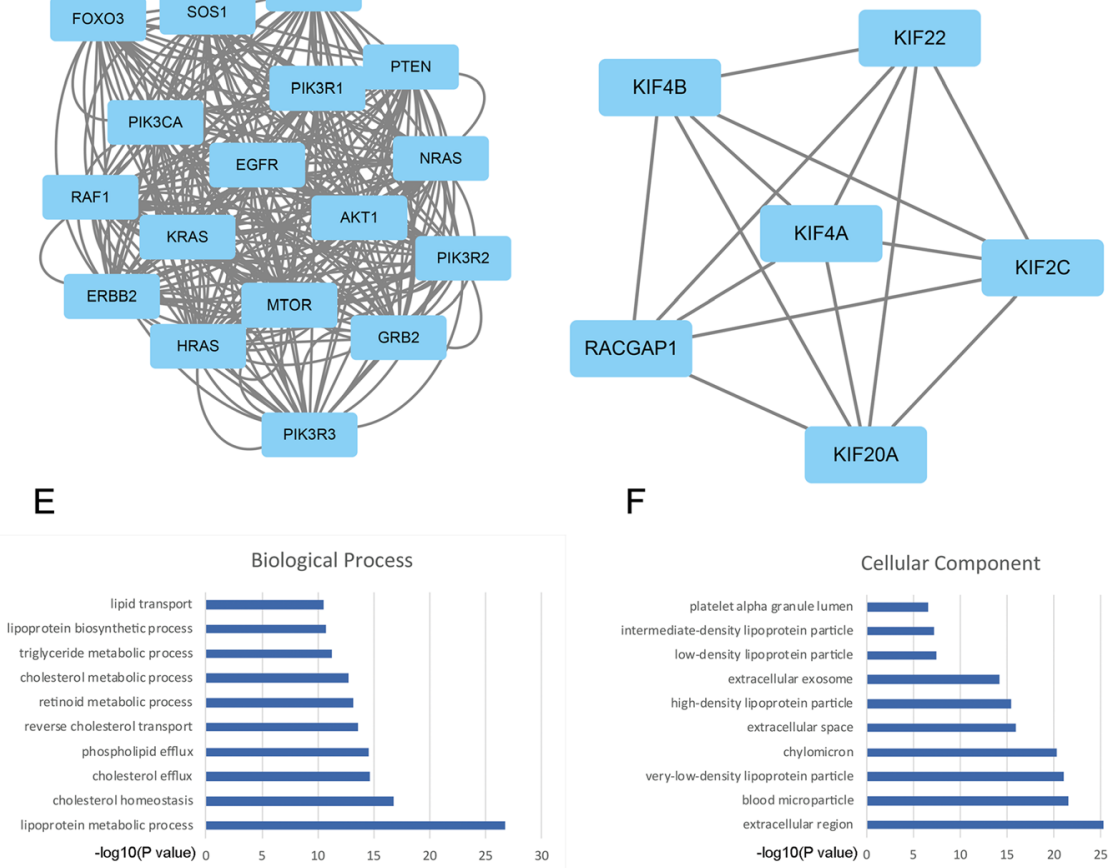

F
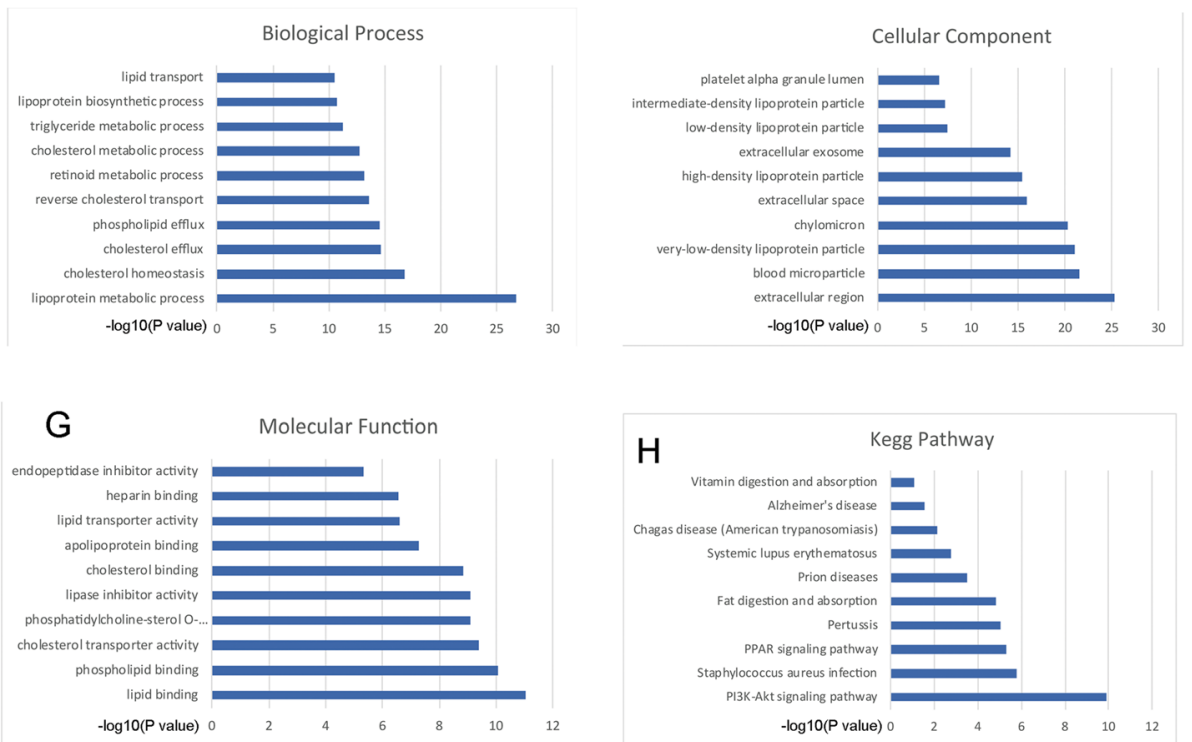

Fig. 2 Protein-protein interaction of Apod and targeting genes (a). MCODE models 1 (b), 2 (c), and 3 (d) of the protein-protein interaction. Biological process (e), cellular component (f), molecular function (g), and the KEGG pathway (h) of the Apod target genes 
interaction (PPI) networks [18]. The PPI networks were mapped using the Cytoscape software (version 3.7.2, http://www.cytoscape.org/). The cutoff criteria for the module genes were set as follows: degree cutoff $\geq 2$, node score cutoff $\geq 2$, K-core $\geq 2$, and max depth $=100$. Subsequently, Gene Ontology (GO) and KEGG pathway enrichment analysis of target genes were conducted via the Database of Annotation, Visualization, and Integration Discovery (DAVID; version 6.8; http://david.abcc. ncifcrf.gov/). GO terms include biological process (BP), cellular component (CC), and molecular function (MF).

\section{Statistical analyses}

Data were expressed as mean \pm standard deviation (SD). SPSS 20.0 software (IBM, Armonk, NY, USA) was applied for data analysis. The $t$ test and one-way ANOVA (followed by Tukey's post hoc test) was used for analyzing the differences between two groups and multiple groups, respectively. $P<0.05$ indicated a significant difference.

\section{Results}

Apod level was upregulated during osteogenesis

Compared with osteogenesis induced in MSCs for 1 day, after osteogenesis induced in MSCs for 3, 7, and 14 days, Apod (Fig. 1a), RUNX2 (Fig. 1b), ALP (Fig. 1c), and Osterix (Fig. 1d) was time-dependently upregulated. Those results indicated that Apod was involved in the osteogenic differentiation of MSCs.

What is more, Western blot was performed and revealed that after osteogenesis is induced in MSCs for 1, 3, 7, and 14 days, Apod was time-dependently upregulated (Fig. 1e), suggesting the involvement of Apod in osteogenesis.

\section{Bioinformatic analysis}

PPI was performed and found that Apod is the center of the total PPI. Then, we used MCODE to screen the modules of

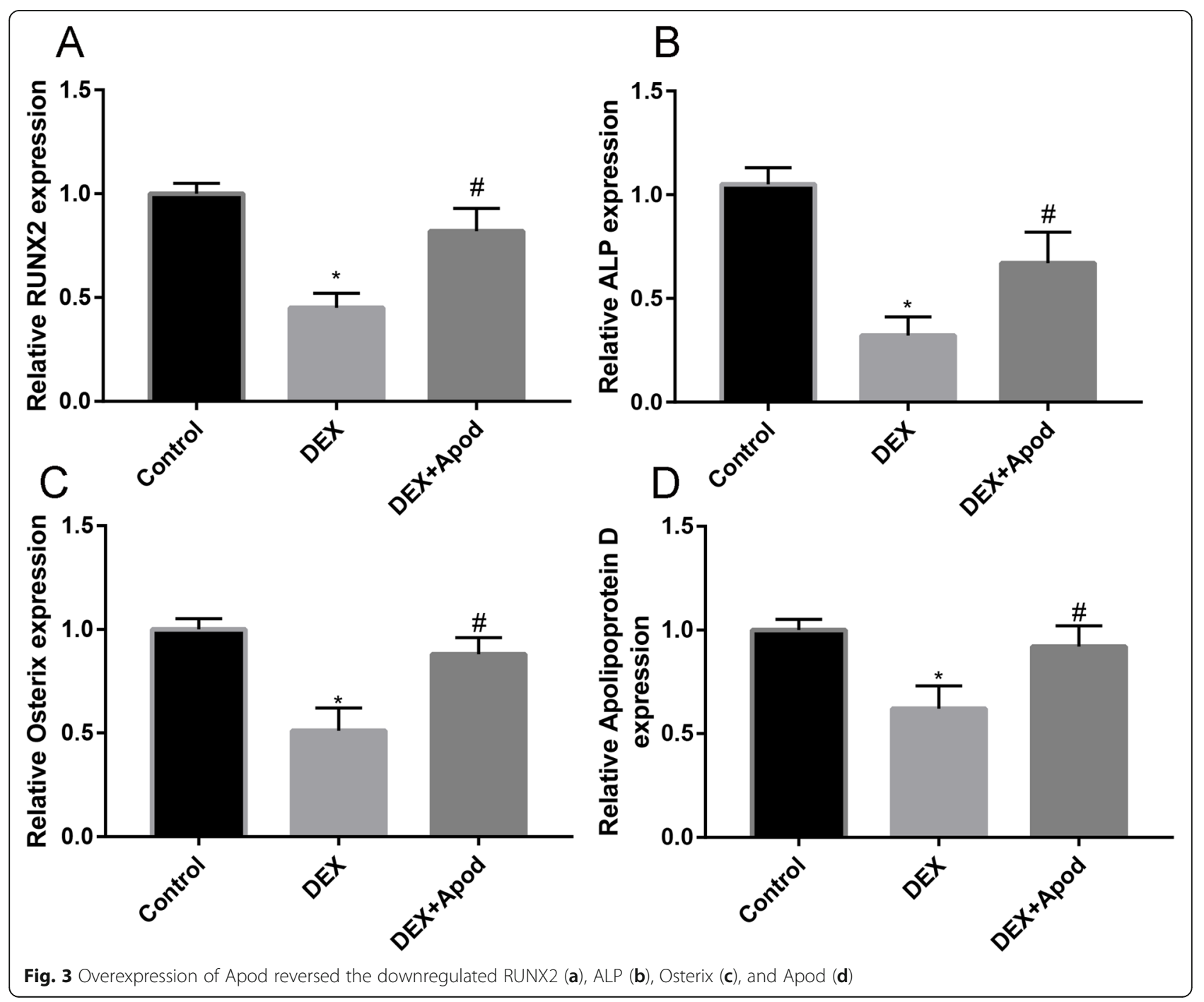


the gene interaction network, and three modules were shown in Fig. $2 \mathrm{a}-\mathrm{d}$. The score of the top 1 module includes FGA, APP, AFP, APOA5, and APOA2. The score of the top 2 module mainly includes PI3CA, EGFR, Akt1, PI3KR2, and PI3KR3. The score of the top 3 module includes KIF4B, KIF4A, KIF22, KIF2C, KIF20A, and RACGAP1.
The functional annotation of the target genes was clarified using the DAVID 6.8 online tool. Biological process analysis indicated that these target genes were significantly enriched in lipoprotein metabolic process, cholesterol homeostasis, and cholesterol efflux (Fig. 2d). For cellular components, the target genes were enriched

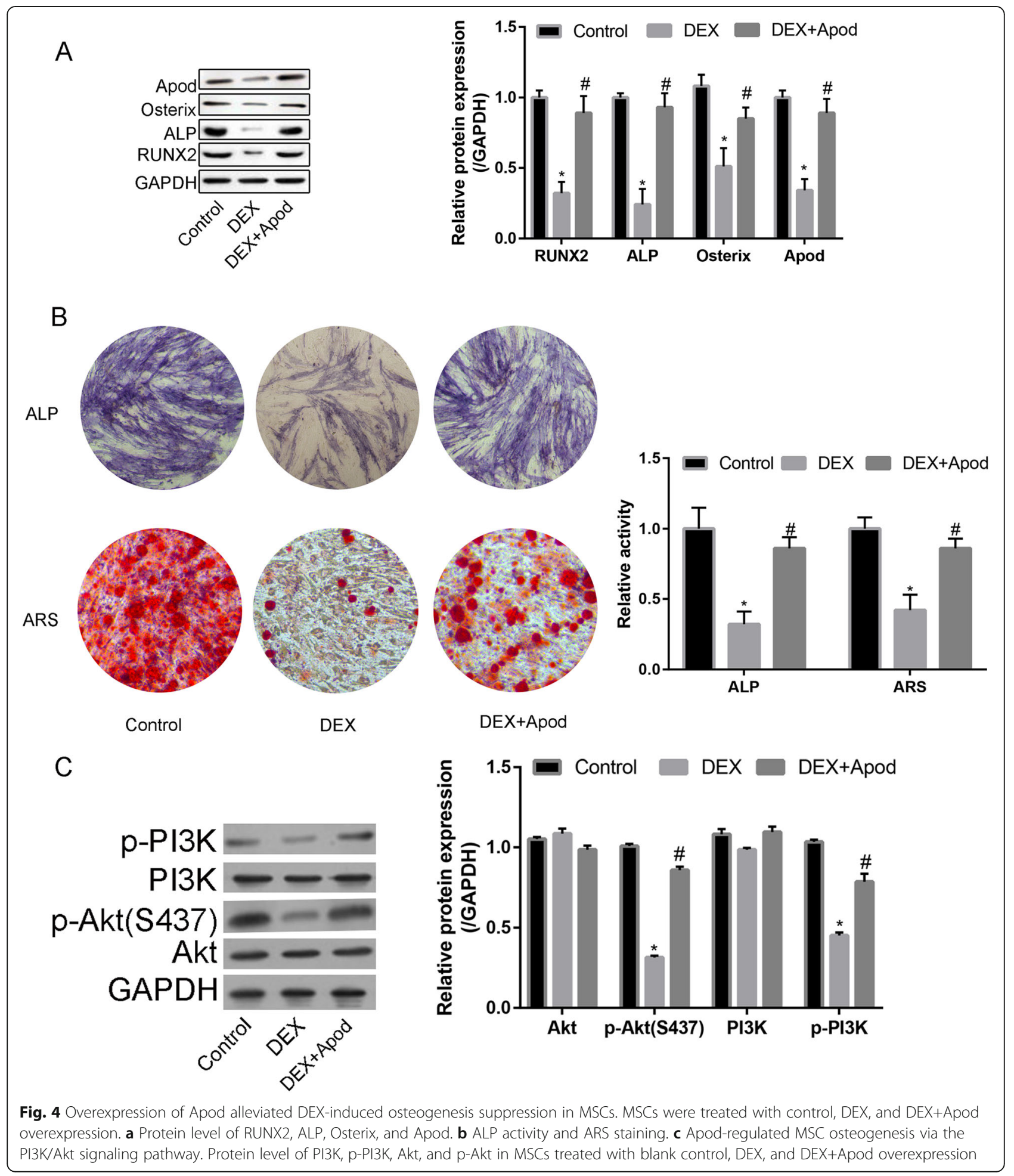


in the extracellular region, blood microparticle, and very low-density lipoprotein particle (Fig. 3e). For molecular function, target genes were significantly enriched in lipid binding, phospholipid binding, and cholesterol transporter activity (Fig. 3f). For the KEGG pathway, target genes mainly enriched in the PI3K-Akt signaling pathway (Fig. 3g).

\section{Overexpression of Apod reversed the downregulated osteogenesis-associated genes in DEX-induced MSCs}

To elucidate the potential function of Apod in MSC osteogenesis, cells were induced in medium containing $1 \mu \mathrm{M}$ DEX. DEX induction markedly downregulated relative levels of RUNX2 (Fig. 3a), ALP (Fig. 3b), and Osterix (Fig. 3c), which were partially reversed by the overexpression of Apod. Moreover, we found that DEX induction markedly downregulated relative levels of Apod, and overexpression of Apod significantly upregulated the expression of Apod (Fig. 3d), which indicated that the cell transfection was successful.

\section{Overexpression of Apod alleviated DEX-induced osteogenesis suppression in MSCs}

The downregulated protein level of RUNX2, ALP, Osterix, and Apod after DEX treatment was reversed by the overexpression of Apod in MSCs, which was similar to its changing trend at the mRNA level (Fig. 4a).

In addition, ALP and ARS activity decreased following DEX treatment, and it was further elevated by overexpression of Apod (Fig. 4b).

\section{Apod regulated MSC osteogenesis via the Wnt pathway} After DEX treatment, protein levels of p-PI3K and p-Akt (S437) were downregulated. However, their downregulation was reversed by the overexpression of Apod (Fig. 4c). It is believed that Apod alleviated GC-induced osteogenesis suppression in MSCs.

\section{Discussion}

GC is one of the most commonly applied drugs. However, the long-term application of GC would lead to many complications, including OP, osteonecrosis, and metabolic syndrome $[19,20]$. GIOP is the most common subtype of $\mathrm{OP}$ and is a metabolic skeletal disorder [21]. However, the mechanism of GIOP was not fully understood.

In our analysis, Apod was upregulated during osteogenesis and accelerated osteogenesis in MSCs. DEX significantly reduced ALP and ARS activity; these effects were reversed by extra adding Apod. Those results found that Apod could regulate the osteogenic differentiation of MSCs. Martineau et al. [9] revealed that when Apod was overexpressed, the osteogenic differentiation ability was increased. Oxidative damage plays a vital role in inducing GIOP. Previous studies found that superoxide dismutase (SOD) and catalase were increased during osteogenic differentiation of hBMSC. ApoD was increased in response to oxidative stress in the brain and has been suggested to function as an antioxidant in the brain [22].

Apod emerges as an evolutionarily conserved antistress protein that is induced by oxidative stress and inflammation and may prove to be an effective therapeutic agent against a variety of neuropathologies, and even against aging [23]. What is more, oxidative stress induces Apod overexpression in the hippocampus during aging and Alzheimer's disease [24].

Moreover, we used bioinformatic analysis to reveal the potential mechanism of Apod in regulating the osteogenic differentiation of MSCs. We found that target genes of Apod mainly enriched in lipoprotein metabolic process, and the KEGG pathway revealed that the PI3K/ Akt signaling pathway was the most enriched pathway. DEX could significantly reduce the p-PI3K and p-Akt expression, while Apod could reverse the downregulation of p-PI3K and p-Akt. Taken together, Apod could reverse the osteogenic inhibitory effect of DEX on osteogenic differentiation of MSCs through the PI3K/Akt signaling pathway.

Our findings uncovered that downregulated osteogenesisassociated genes in DEX-induced MSCs were partially reversed by the overexpression of Apod, as well as the attenuated ALP activity and mineralization ability. Notably, Apod overexpression activated the PI3K/Akt pathway in DEXinduced MSCs.

\section{Conclusions}

Apod alleviates glucocorticoid-induced osteogenesis suppression in MSCs via the PI3K/Akt pathway, thus protecting the progression of GIOP.

\section{Abbreviations \\ Apod: Apolipoprotein D; MSCs: Mesenchymal stem cells; OP: Osteoporosis; DEX: Dexamethasone; GIOP: Glucocorticoid-induced osteoporosis; GC: Glucocorticoid; DMEM: Dulbecco's modified Eagle's medium; FBS: Fetal bovine serum; q-RTPCR: Quantitative reverse transcriptase-polymerase chain reaction assay; SDS-PAGE: Sodium dodecyl sulfate-polyacrylamide gel electro- phoresis; HRP: Horseradish peroxidase; ALP: Alkaline phosphatase; ARS: Alizarin red staining; STRING: Search Tool for the Retrieval of Interacting Genes; MCODE: Molecular Complex Detection; PPI: Protein-protein interaction; DAVID: Database of Annotation, Visualization, and Integration Discovery; BP: Biological process; CC: Cellular component; MF: Molecular function; SPSS: Statistical Product and Service Solutions; SOD: Superoxide dismutase}

\section{Acknowledgements}

Not applicable.

Authors' contributions

Rong-Hua Yu and Xiang-yang Zhang designed the study. Wei Xu and Zhi-kun L performed the study. Xiao-dong Zhu and Zhi-kun Li supervised the experiment. All authors approved the final study. 


\section{Funding}

This work was supported by the National Natural Science Foundation of China (No. 81903039), the Sponsored by Shanghai Sailing Program (No. 19YF1444500), the Excellent young medical talents training plan of the Shanghai Health Planning Commission (No. 2018YQ46), the Changning District Committee of Science and Technology (No. CNKW2017Y07), the Changning District Health and Family Planning Commission (No. 20164Y003, 20194Y008), and the Sixth People's Hospital of Shanghai Medical Group Projects (No. ly201802, ly201903)

\section{Availability of data and materials}

The datasets used and/or analyzed during the current study are available from the corresponding authors upon reasonable request.

\section{Ethics approval and consent to participate}

This study was approved and consented to participate by the Ethics Committee.

\section{Consent for publication}

Not applicable.

\section{Competing interests}

The authors declare that they have no competing interests.

Received: 22 February 2020 Accepted: 29 July 2020

Published online: 08 August 2020

\section{References}

1. Zheng LZ, Wang $J$, Kong L, Huang L, Tian L, Pang QQ, Wang XL, Qin L. Steroid-associated osteonecrosis animal model in rats. J Orthop Translat. 2018:13:13-24

2. Xie $X H$, Wang $X L$, Yang HL, Zhao DW, Qin L. Steroid-associated osteonecrosis: epidemiology, pathophysiology, animal model, prevention, and potential treatments (an overview). J Orthop Translat. 2015;3:58-70.

3. Xie $X H$, Wang $X L$, He YX, Liu Z, Sheng $H$, Zhang G, Oin L. Promotion of bone repair by implantation of cryopreserved bone marrow-derived mononuclear cells in a rabbit model of steroid-associated osteonecrosis. Arthritis Rheum. 2012;64:1562-71.

4. Buckley L, Humphrey MB. Glucocorticoid-induced osteoporosis. N Engl J Med. 2018:379:2547-56.

5. He X, Jittiwat J, Kim JH, Jenner AM, Farooqui AA, Patel SC, Ong WY Apolipoprotein D modulates F2-isoprostane and 7-ketocholestero formation and has a neuroprotective effect on organotypic hippocampal cultures after kainate-induced excitotoxic injury. Neurosci Lett. 2009:455: 183-6.

6. Belloir B, Kovari E, Surini-Demiri M, Savioz A. Altered apolipoprotein D expression in the brain of patients with Alzheimer disease. J Neurosci Res. 2001;64:61-9.

7. Ordonez C, Navarro A, Perez C, Astudillo A, Martinez E, Tolivia J. Apolipoprotein $D$ expression in substantia nigra of Parkinson disease. Histol Histopathol. 2006;21:361-6.

8. Rassart E, Bedirian A, Do Carmo S, Guinard O, Sirois J, Terrisse L, Milne R. Apolipoprotein D. Biochim Biophys Acta. 2000;1482:185-98.

9. Martineau C, Najyb O, Signor C, Rassart E, Moreau R. Apolipoprotein D deficiency is associated to high bone turnover, low bone mass and impaired osteoblastic function in aged female mice. Metabolism. 2016;65: 1247-58.

10. Ishii M, Koike C, Igarashi A, Yamanaka K, Pan H, Higashi Y, Kawaguchi H, Sugiyama M, Kamata N, Iwata T, Matsubara T, Nakamura K, Kurihara H, Tsuj K, Kato Y. Molecular markers distinguish bone marrow mesenchymal stem cells from fibroblasts. Biochem Biophys Res Commun. 2005:332:297-303.

11. Beak JY, Kang HS, Kim YS, Jetten AM. Kruppel-like zinc finger protein Glis3 promotes osteoblast differentiation by regulating FGF18 expression. J Bone Miner Res. 2007:22:1234-44.

12. Schilling AF, Schinke T, Munch C, Gebauer M, Niemeier A, Priemel M, Streichert T, Rueger JM, Amling M. Increased bone formation in mice lacking apolipoprotein E. J Bone Miner Res. 2005;20:274-82.

13. Zhao Z, Ma X, Ma J, Sun X, Li F, Lv J. Naringin enhances endothelia progenitor cell (EPC) proliferation and tube formation capacity through the CXCL12/CXCR4/PI3K/Akt signaling pathway. Chem Biol Interact. 2018;286: $45-51$.
14. Ye C, Zhang W, Hang K, Chen M, Hou W, Chen J, Chen X, Chen E, Tang L, Lu J, Ding Q, Jiang G, Hong B, He R. Extracellular IL-37 promotes osteogenic differentiation of human bone marrow mesenchymal stem cells via activation of the PI3K/AKT signaling pathway. Cell Death Dis. 2019;10:753.

15. Mukherjee A, Rotwein P. Akt promotes BMP2-mediated osteoblast differentiation and bone development. J Cell Sci. 2009:122:716-26.

16. Srivastava S, Sharma K, Kumar N, Roy P. Bradykinin regulates osteoblast differentiation by Akt/ERK/NFkB signaling axis. J Cell Physiol. 2014;229:2088-105.

17. Ulici $V$, Hoenselaar KD, Gillespie JR, Beier F. The PI3K pathway regulates endochondral bone growth through control of hypertrophic chondrocyte differentiation. BMC Dev Biol. 2008:8:40.

18. Bandettini WP, Kellman P, Mancini C, Booker OJ, Vasu S, Leung SW, Wilson JR, Shanbhag SM, Chen MY, Arai AE. MultiContrast Delayed Enhancement (MCODE) improves detection of subendocardial myocardial infarction by late gadolinium enhancement cardiovascular magnetic resonance: a clinical validation study. J Cardiovasc Magn Reson. 2012;14:83.

19. Weston JM, Norris EV, Clark EM. The invisible disease: making sense of an osteoporosis diagnosis in older age. Qual Health Res. 2011;21:1692-704.

20. Sandhu SK, Hampson G. The pathogenesis, diagnosis, investigation and management of osteoporosis. J Clin Pathol. 2011;64:1042-50.

21. Lane NE. Epidemiology, etiology, and diagnosis of osteoporosis. Am J Obstet Gynecol. 2006;194:S3-11.

22. Ganfornina MD, Do Carmo S, Lora JM, Torres-Schumann S, Vogel M, Allhorn M, Gonzalez C, Bastiani MJ, Rassart E, Sanchez D. Apolipoprotein D is involved in the mechanisms regulating protection from oxidative stress. Aging Cell. 2008;7:506-15.

23. Dassati S, Waldner A, Schweigreiter R. Apolipoprotein D takes center stage in the stress response of the aging and degenerative brain. Neurobiol Aging. 2014;35:1632-42

24. Martinez E, Navarro A, Ordonez C, Del Valle E, Tolivia J. Oxidative stress induces apolipoprotein D overexpression in hippocampus during aging and Alzheimer's disease. J Alzheimers Dis. 2013;36:129-44.

\section{Publisher's Note}

Springer Nature remains neutral with regard to jurisdictional claims in published maps and institutional affiliations.

Ready to submit your research? Choose BMC and benefit from:

- fast, convenient online submission

- thorough peer review by experienced researchers in your field

- rapid publication on acceptance

- support for research data, including large and complex data types

- gold Open Access which fosters wider collaboration and increased citations

- maximum visibility for your research: over $100 \mathrm{M}$ website views per year

At BMC, research is always in progress.

Learn more biomedcentral.com/submissions 